\title{
Broadband Circular Polarizer Based on Plasmon Hybridizations
}

\author{
Hong-Yu Shi, ${ }^{1}$ Wei Li, ${ }^{2}$ Shi-Tao Zhu, ${ }^{2}$ Xiao-Li Dong, ${ }^{3}$ An-Xue Zhang, ${ }^{1,4}$ \\ Song $\mathrm{Xia}^{2}$ and $\mathrm{Zhuo} \mathrm{Xu}^{2}$ \\ ${ }^{1}$ School of Electronic and Information Engineering, Xian Jiaotong University, Xian 710049, China \\ ${ }^{2}$ Electronic Materials Research Laboratory, Key Laboratory of the Ministry of Education, Xian Jiaotong University, \\ Xian 710049, China \\ ${ }^{3}$ School of Telecommunication and Information Engineering, Xian University of Posts and Telecommunications, Xian 710061, China \\ ${ }^{4}$ Beijing Center for Mathematics and Information Interdisciplinary Science (BCMIIS), Beijing 100048, China
}

Correspondence should be addressed to An-Xue Zhang; anxuezhang@mail.xjtu.edu.cn

Received 5 January 2017; Revised 11 February 2017; Accepted 20 February 2017; Published 7 March 2017

Academic Editor: Vincenzo Galdi

Copyright (C) 2017 Hong-Yu Shi et al. This is an open access article distributed under the Creative Commons Attribution License, which permits unrestricted use, distribution, and reproduction in any medium, provided the original work is properly cited.

This paper presents a broadband circular polarizer with a ring/disk cavity structure, which is a broadband application of plasmon hybridizations. The proposed design can convert a linearly polarized wave to a circularly polarized wave from $12.66 \mathrm{GHz}$ to $17.43 \mathrm{GHz}$ within a bandwidth of $30 \%$. The broadband polarization conversion characterization results from the different plasmon hybridization modes induced in the ring/disk cavity. The proposed broadband circular polarizer is demonstrated by both full-wave simulation and measurement.

\section{Introduction}

Metamaterials are artificial materials with properties which do not exist in natural material. They have been broadly used to control properties of the electromagnetic wave [1-3], especially the polarization [4-7]. Numerous metamaterialinspired polarizers have been designed to change the polarization properties of antennas [8-12].

Plasmon resonances and plasmon hybridizations have been observed in metallic metamaterial structures [13, 14]. Plasmon hybridizations, including Fano resonances, can be induced within complex structures supporting multiple plasmon resonances even at microwave wavelengths. Fedotov et al. observed Fano resonance in a planar metallic metamaterial structure by introducing symmetry breaking [15]. These interesting phenomena have been applied for electromagnetic waves control, such as polarization converter $[16,17]$, absorber [18], and wave guiding and negative refraction [19, 20].

Metallic rings are highly tunable structures which can support multipolar plasmon modes at different frequencies under oblique incidence, retardation effects, or coupling with a disk [21]. Even and odd cavity resonant modes have been observed in elliptical plasmonic nanoantennas by azimuthal symmetry breaking, and these resonant modes have been applied to realize polarization conversion and absorption with a relatively low efficiency [22]. Using symmetry breakings, Fano resonance can also be raised in nonconcentric ring/disk cavities by the interaction between higher multipolar modes [23, 24]. Recently, various plasmon hybridization modes have been observed in highly tunable concentric ring/disk cavities at microwave band [25].

Khanikaev et al. designed an efficient ultra-thin circular polarizer using plasmon hybridizations in a unit cell with two topologically distinct structures. However, it only works at a single frequency [26]. Zhu et al. proposed a circular polarizer using electromagnetically induced transparency like (EITlike) effect raised by two pairs of cut wires in the microwave band. However, the bandwidth of this design is only about $5.5 \%$ (from $9 \mathrm{GHz}$ to $9.5 \mathrm{GHz}$ ) [27]. In this paper, a broadband circular polarizer is proposed using plasmon hybridizations in a ring/disk cavity. Different polarization selective plasmon hybridizations can be generated in the ring/disk cavity and cause a broadband circular polarization conversion from $12.66 \mathrm{GHz}$ to $17.43 \mathrm{GHz}$ within a bandwidth of about $30 \%$. 


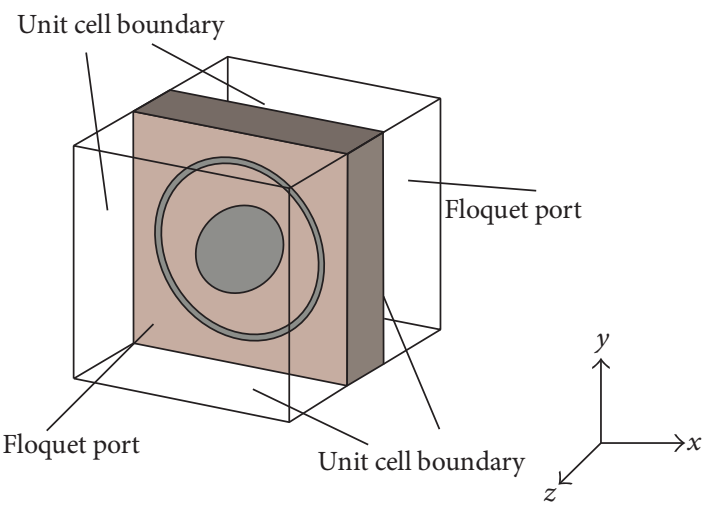

FIGURE 1: Schematic of the simulation.

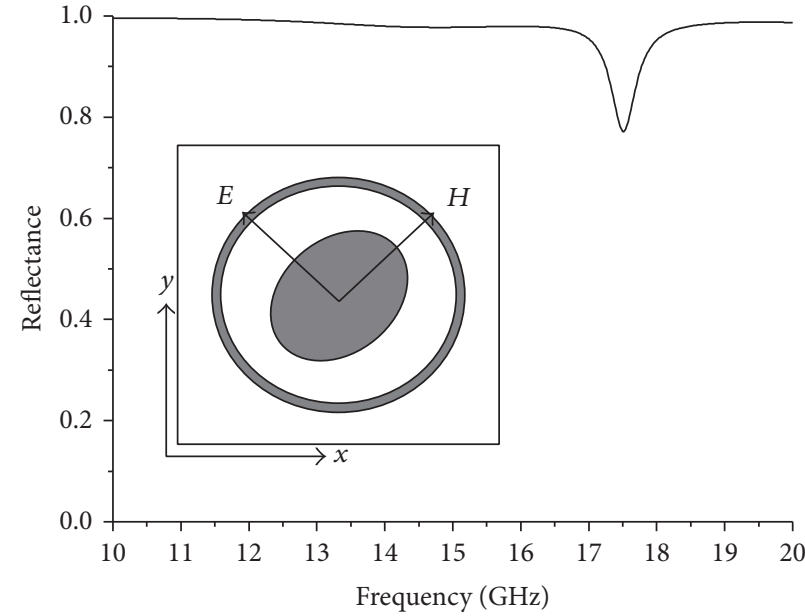

(a)

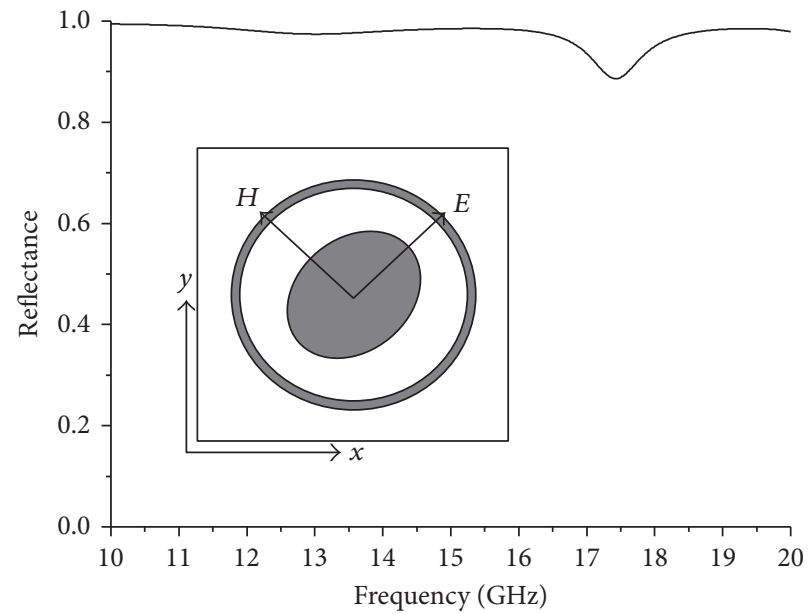

(b)

FIgURE 2: The amplitudes of the reflectance under the illumination of (a) major axis and (b) minor axis polarized waves.

\section{Polarization Selected Plasmon Hybridizations in the Ring/Disk Cavity}

The proposed ring/disk cavity is composed of an elliptical disk and a circular ring. The elliptical disk introduces anisotropic properties to the structure and makes the ring/disk cavity support different plasmon hybridization modes for different polarized incident waves at different frequencies. These plasmon hybridization modes can be observed through the distributions of the electric field Ez within the ring/disk cavity. We build a model in a commercial software CST MICROWAVE STUDIO as shown in Figure 1. In the simulation, the unit cell boundary condition is used along the $x$ and $y$ directions, and the absorbing boundary condition is applied in the $z$ direction. The model is excited by Floquet port with a normal incidence of linearly polarized waves in a frequency range of $10 \mathrm{GHz}-18 \mathrm{GHz}$.

Figure 2 shows the amplitudes of the reflectance under the illumination of waves polarized along the major axis and the minor axis of the elliptical disk. There is a dip at $17.5 \mathrm{GHz}$ under major axis polarized incidence and a dip at $17.4 \mathrm{GHz}$ under minor axis polarized incidence. Because of the metallic back sheet, the transmission is zero; thus, these reflectance dips are totally caused by the absorption. Both reflectances are bigger than 0.8 from $12 \mathrm{GHz}$ to $17.43 \mathrm{GHz}$.

The excited plasmon hybridization modes at different frequencies under major axis and minor axis polarized incidences are shown in Figure 3. In the ring/disk cavity, the different ring modes and disk modes couple to each other and generate different plasmon hybridizations.

Figure 3(a) shows the plasmon hybridizations excited under the major axis polarized incidence. At $12 \mathrm{GHz}$, the dipolar ring mode is out phase with the ell cavity mode [22] of the disk which causes a dark mode in the ring/disk cavity. At $15 \mathrm{GHz}$, the mimicking gap surface plasmons are generated [22] and in phase with the dipolar ring mode. The ring is excited in hexapole ring mode and is out of phase with the ell disk mode at $16.5 \mathrm{GHz}$. At $17.5 \mathrm{GHz}$, the mimicking gap surface plasmons are stronger and in phase with the hexapole ring mode. The disk resonates in e12 mode [22] at 17.5 GHz. The hexapole ring mode, the ell disk mode, and the mimicking gap surface plasmons are in phase with each other at $18 \mathrm{GHz}$.

Under minor axis polarized incidence, the resonances are shown in Figure 3(b). The dipolar ring mode is in phase with the oll disk mode [22] at $12 \mathrm{GHz}, 15 \mathrm{GHz}$, and $16 \mathrm{GHz}$. 

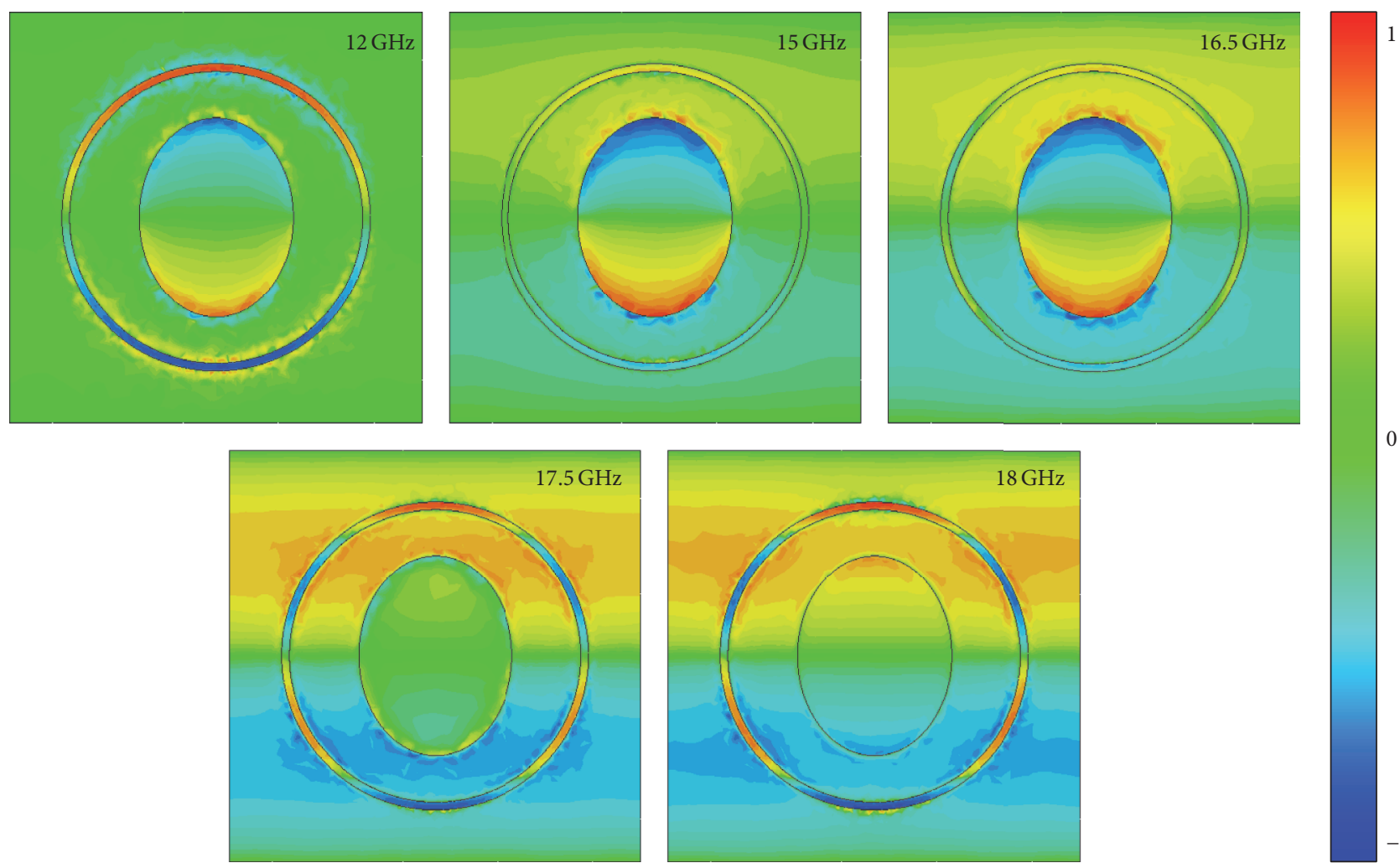

(a)
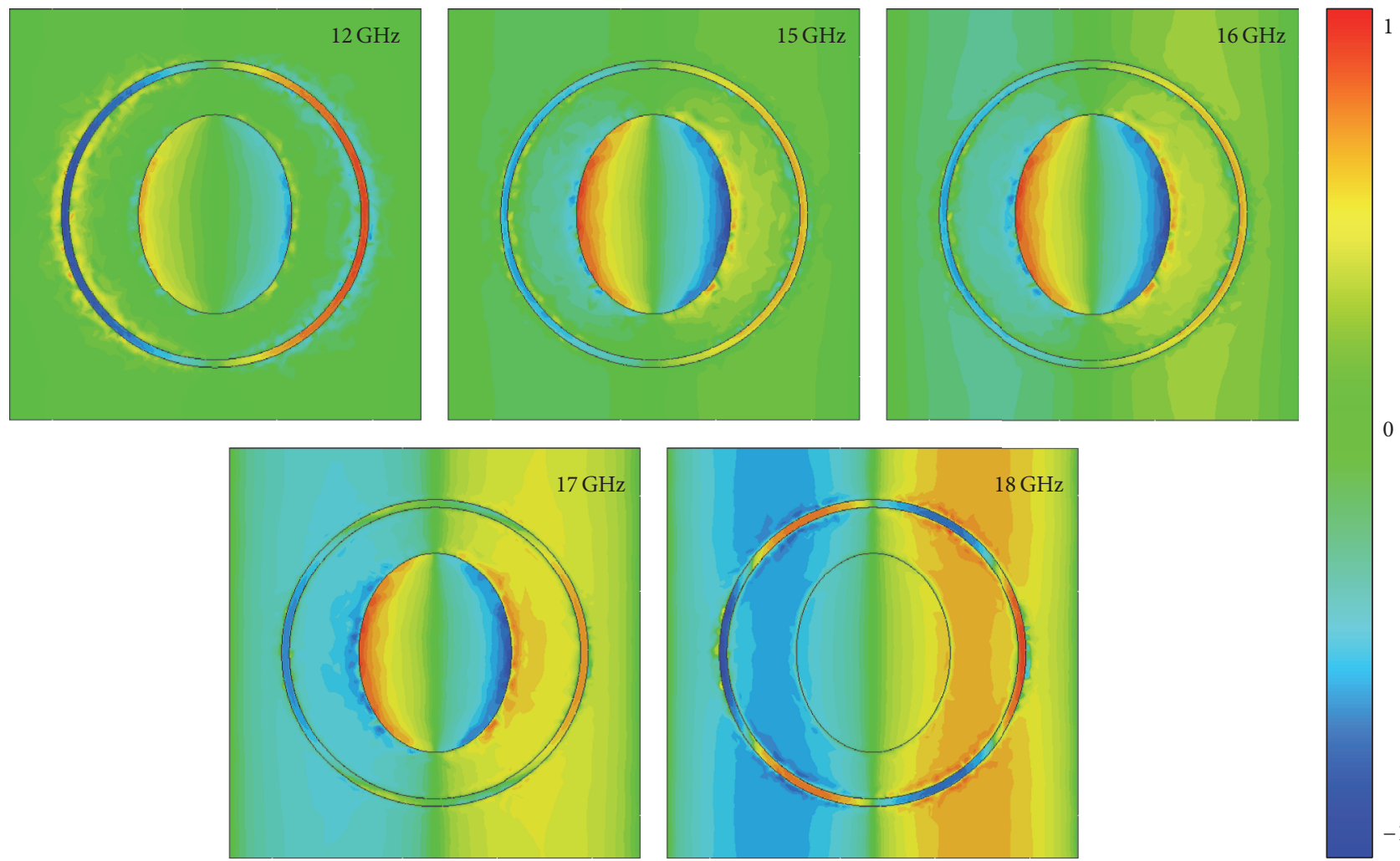

(b)

FIGURE 3: The resonances in the ring/disk cavity: (a) under major axis polarized incidence; (b) under minor axis polarized incidence. 


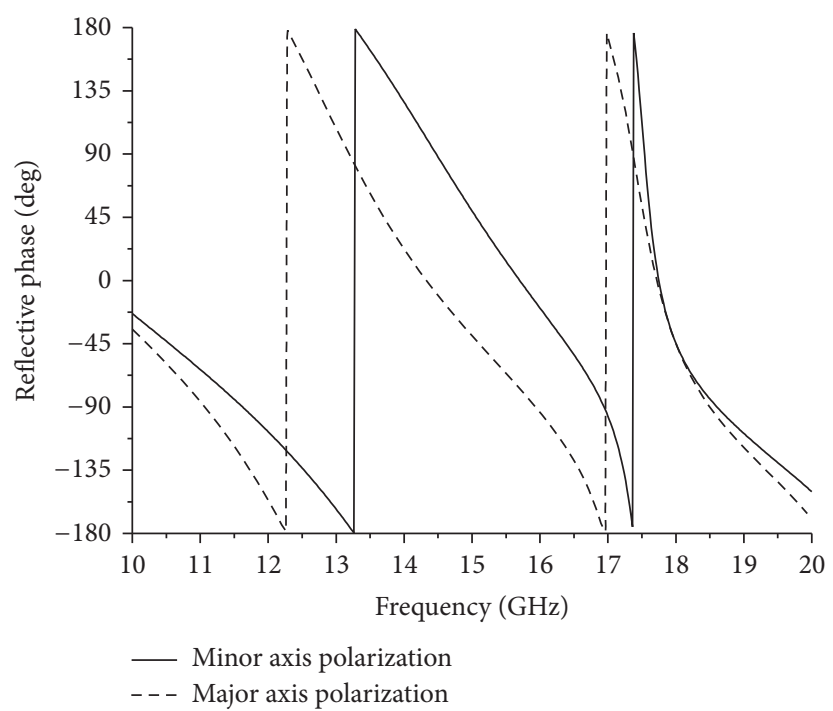

FIGURE 4: The phase of the reflectance under major axis polarized incidence and minor axis polarized incidence, respectively.

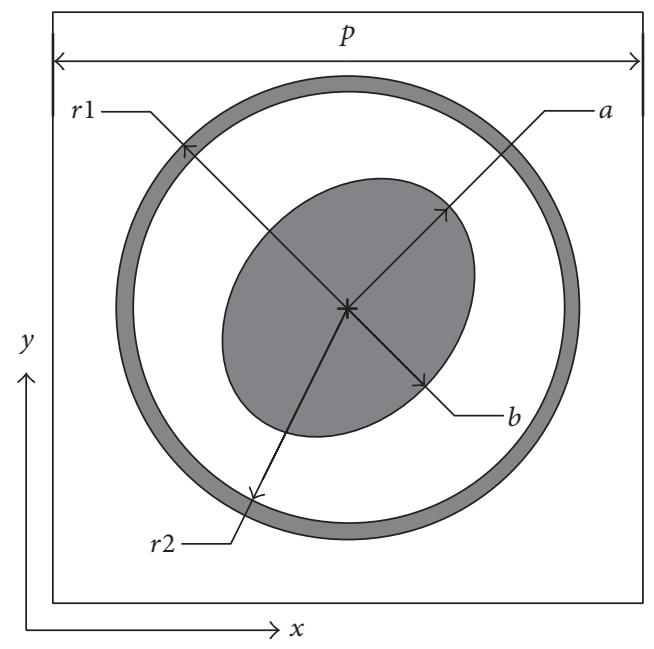

FIGURE 5: The structure of the ring/disk cavity.

The mimicking gap surface plasmons generated from $16 \mathrm{GHz}$ are in phase with the ring modes and stronger at higher frequency. The hexapole ring mode is out phase and in phase with the oll disk mode at $17 \mathrm{GHz}$ and $18 \mathrm{GHz}$, respectively.

Because of the metallic back sheet, the reflectance amplitude of major axis and minor axis polarized components are proximate to each other as shown in Figure 2. However, due to the anisotropic of the ring/disk cavity, the polarization selective plasmon hybridization modes have a strong influence on the reflection phase. As shown in Figure 4, a broadband reflection phase difference is raised between the major axis and minor axis polarized incidences from $12.66 \mathrm{GHz}$ to 17.43 GHz. Thus, the polarization of the total reflected wave can be controlled by tuning the phase difference. If the phase difference is $90 \mathrm{deg}$, the total reflective wave will be converted to a circularly polarized wave within a bandwidth of about $30 \%$.

\section{Design of the Circular Polarizer}

The proposed C2-symmetric ring/disk cavity can be used to realize a broadband circular polarizer as shown in Figure 5. The included angle between $y$-axis and the major axis of the elliptical disk $\varphi$ is $45 \mathrm{deg}$. The structures are printed on a TACONIC RF-35 slab with a thickness of $h=3.18 \mathrm{~mm}$ and a permittivity of 3.5. The loss tangent of the substrate is 0.003 . The designed circular polarizers are etched from $0.035 \mathrm{~mm}$ flat copper on the substrate. A metallic sheet is on the other side of the substrate to confirm the reflection of the incident power.

As shown in the Figure 5, under $x$ - or $y$-polarized incidence, the incident wave can be decomposed into waves polarized along the major axis and the minor axis with the same amplitude and phase. Due to the anisotropy, the ring/disk cavity has different responses for major axis and minor axis polarized components. And the phase of these 


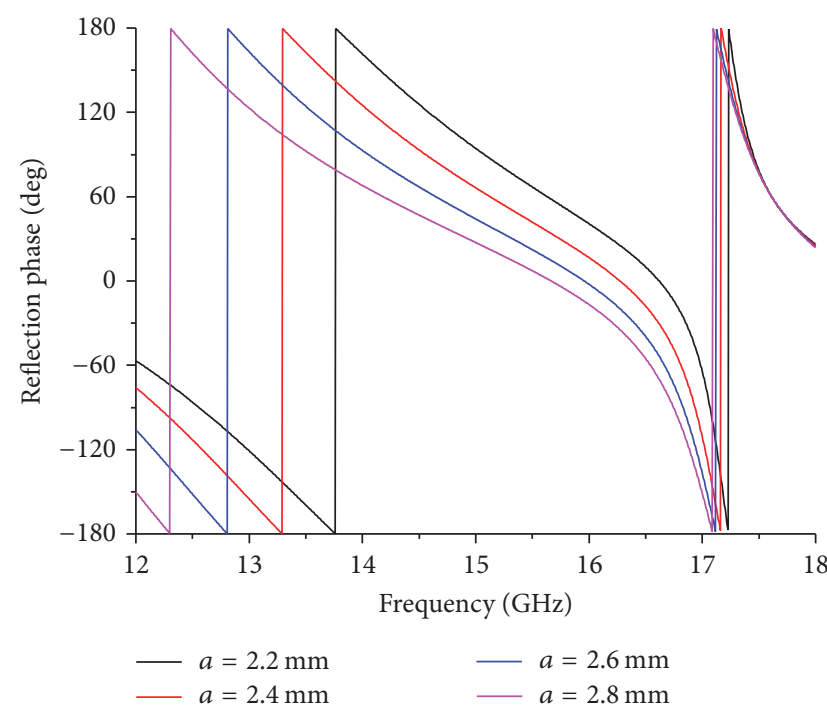

(a)

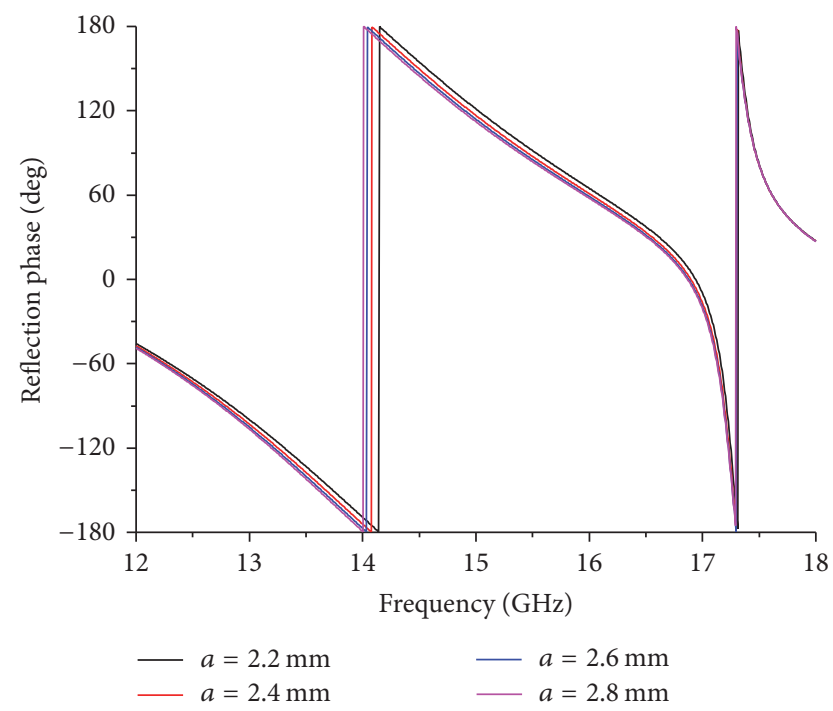

(b)

FIGURE 6: Reflection phase for various values of $a$ : (a) under major axis polarized incidence; (b) under minor axis polarized incidence.

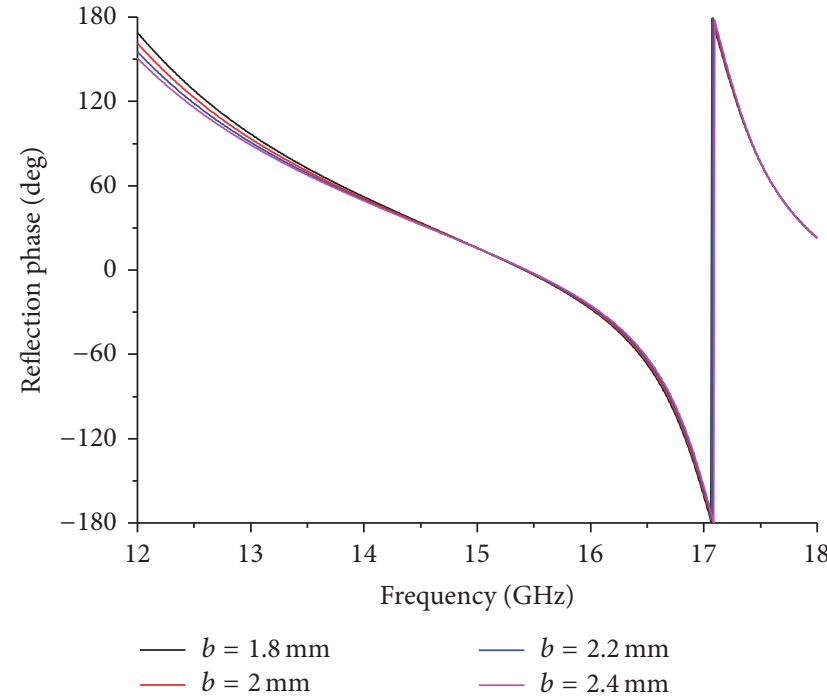

(a)

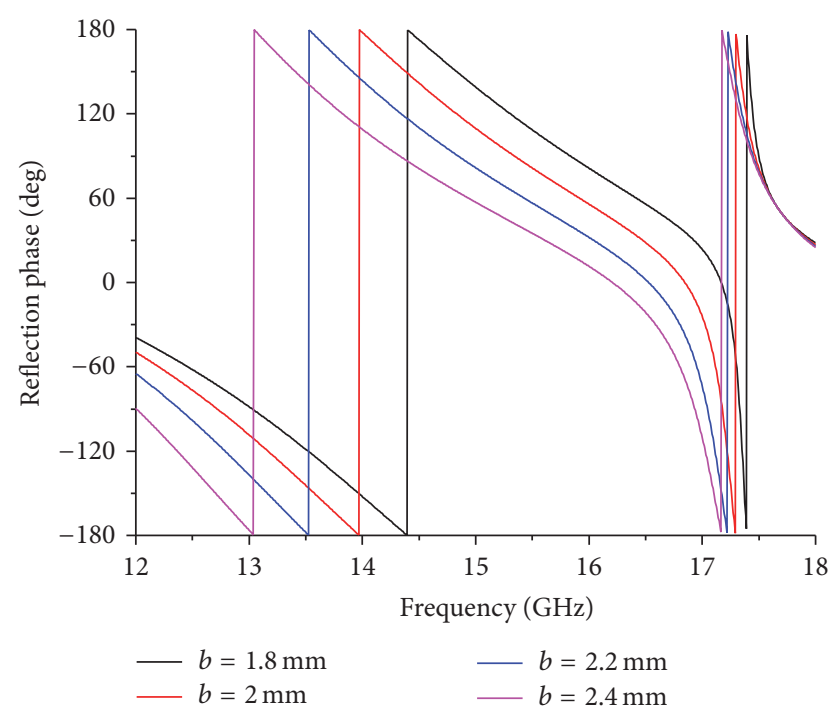

(b)

FIGURE 7: Reflection phase for various values of $b$ : (a) under major axis polarized incidence; (b) under minor axis polarized incidence.

two components can be controlled independently by the geometrical parameters " $a$ " and " $b$." Thus, if the major axis and minor axis polarized components have similar amplitudes and a 90 deg phase difference, the total reflective wave will be circularly polarized.

Figure 6 shows the reflection phase for various values of $a$. As shown in Figure 6(a), the reflection phase under major axis polarized incidence is larger with a smaller $a$. However, the parameter $a$ has a negligible impact on the reflection phase under minor axis polarized incidence, as shown in Figure 6(b).

As shown in Figure 7(a), the reflection phase can be hardly controlled by the values of $b$ under major axis polarized incidence. However, parameter $b$ has a strong impact on the reflection phase under minor axis polarized incidence, as shown in Figure 7(b). With a bigger $b$, the reflection phase under major axis polarized incidence is smaller. Both $a$ and $b$ have a slight influence on the reflection phase at frequencies above $17.3 \mathrm{GHz}$.

As shown in Figure $8, r 1$ has a strong impact on the reflection phase at higher frequencies under both major and minor axis polarized incidences. With bigger $r 1$, the reflection phase is smaller. Below $16.7 \mathrm{GHz}, r 1$ have little influence on the reflection phase, especially under major axis polarized incidence.

As shown in Figure 9, $r 2$ only has a negligible impact on the reflection phase under both major and minor axis polarized incidences. 


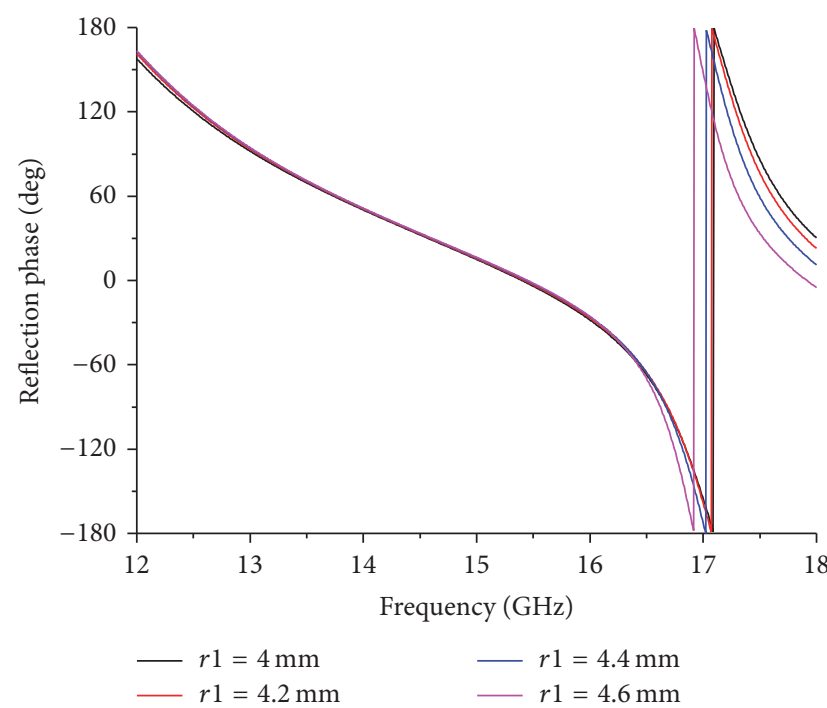

(a)

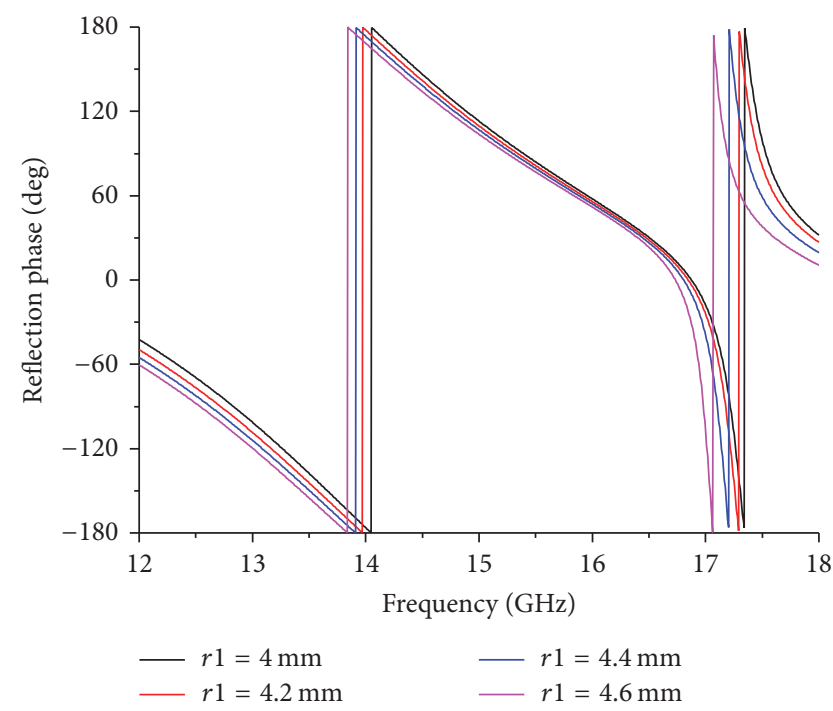

(b)

FIGURE 8: Reflection phase for various values of $r$ : (a) under major axis polarized incidence; (b) under minor axis polarized incidence.

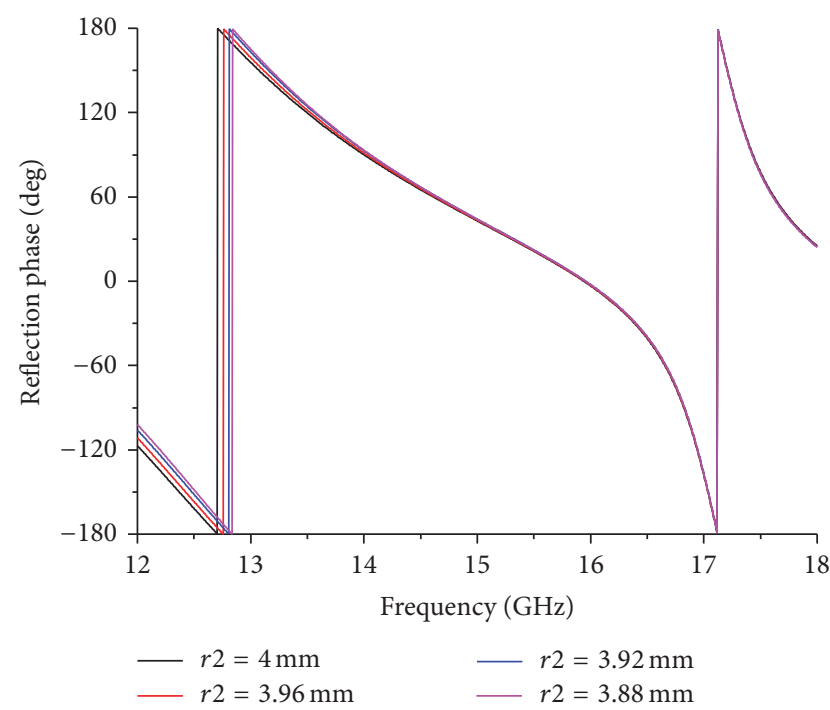

(a)

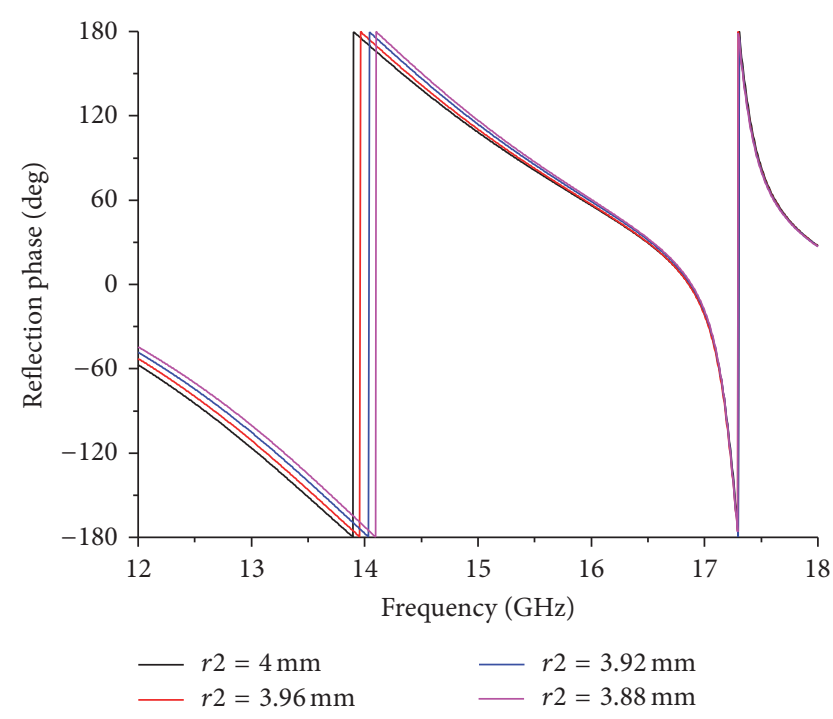

(b)

FIGURE 9: Reflection phase for various values of $r$ : (a) under major axis polarized incidence; (b) under minor axis polarized incidence.

With careful investigation, the geometrical parameters of the unit cell are chosen as $p=10.7 \mathrm{~mm}, r 1=4.2 \mathrm{~mm}, r 2=$ $3.92 \mathrm{~mm}, a=2.6 \mathrm{~mm}$, and $b=2 \mathrm{~mm}$ to obtain a 90 deg phase difference between major and minor axis polarized incidence.

\section{The Simulation and Measurement Results}

Due to the C2-symmetry of the structure, the copolarized and cross-polarized reflected wave are of the same amplitude but with an opposite phase difference under $x$ - or $y$-polarized incidence. Thus, the helicity of the reflective wave is opposite with $x$ - and $y$-polarized illumination.

The simulated amplitude of the reflection coefficient is shown in Figure 10. $\left|R_{i j}\right|$ refers to the amplitude of the $i$-polarized component of the reflective wave when $j$ polarized wave incidents with unit power, where the subscripts $i$ and $j$ could be $x$ or $y$. From $12.66 \mathrm{GHz}$ to $17.43 \mathrm{GHz}$, the amplitude of the $y$ - and $x$-component of the reflective wave fluctuates around 0.7 within a limited range, under $y$ polarized incidence. The central frequency is about $15 \mathrm{GHz}$, and the relative bandwidth is about $30 \%$. Figure 11 shows the 


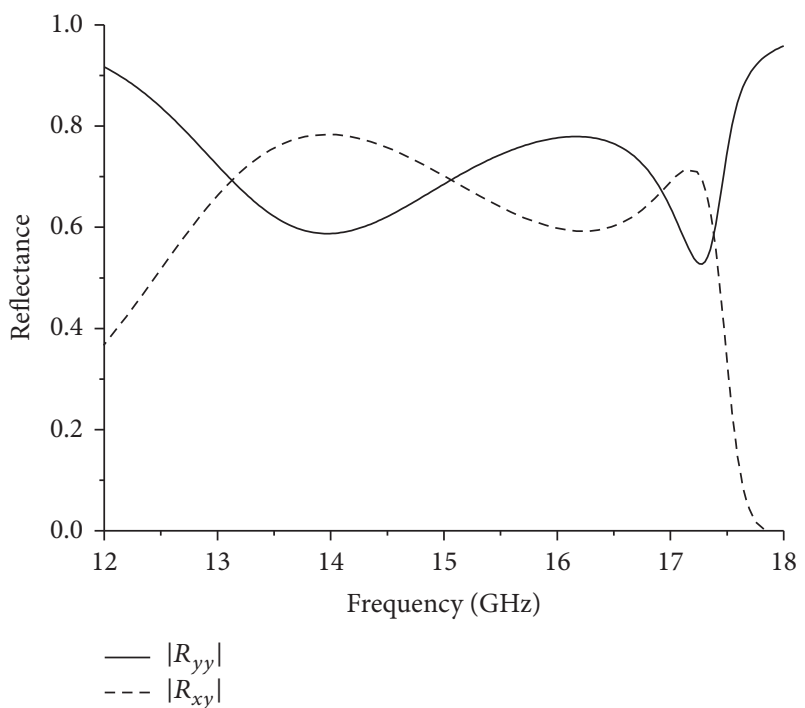

Figure 10: The simulated reflectance of the broadband circular polarizer.

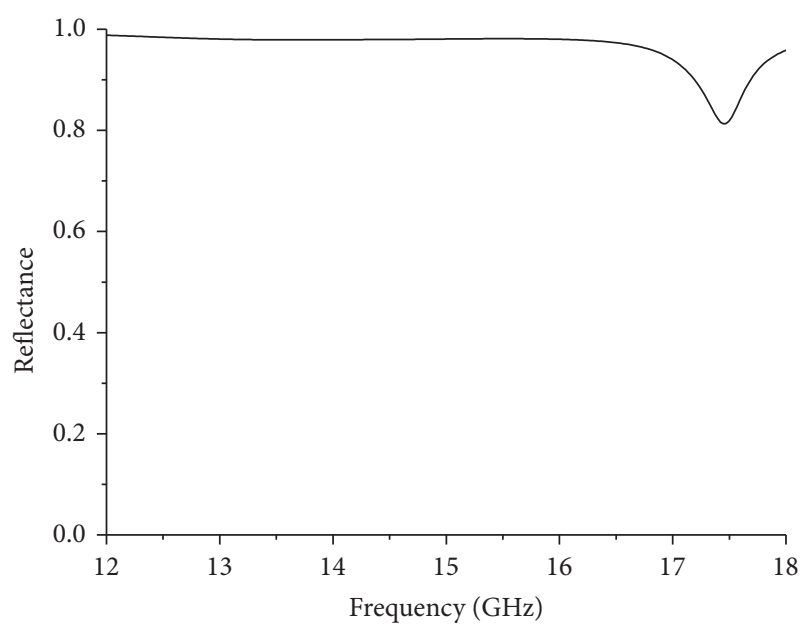

Figure 11: The total reflection amplitude.

total reflection amplitude calculated by $\sqrt{R_{y y}^{2}+R_{x y}^{2}}$. From $12.66 \mathrm{GHz}$ to $17.43 \mathrm{GHz}$, the total reflection amplitude is above 0.8 .

The phase difference of the $x$ - and $y$-component of the reflective wave under $y$-polarized incidence is shown in Figure 12. From $12 \mathrm{GHz}$ to $17.43 \mathrm{GHz}$, the phase difference of the $x$-and $y$-component of the reflective wave is about $90 \mathrm{deg}$. Thus, from $12.6 \mathrm{GHz}$ to $17.43 \mathrm{GHz}$, the reflective wave is lefthanded circularly polarized.

For a further insight of the reflective wave, the ellipticity of the reflective wave is calculated by $\eta=$ $(1 / 2) \sin ^{-1}\left\{2 R \sin (\delta) /\left(1+R^{2}\right)\right\}$, where $\delta$ and $R=\left|R_{x y}\right| /\left|R_{y y}\right|$ are the phase difference and amplitude ratio of the $x$ - and $y$-component of the reflective wave, respectively. When the ellipticity is negative, the reflective wave is left-handed circularly or elliptically polarized. The reflective wave is pure left-handed circularly wave if the ellipticity is $-45 \mathrm{deg}$.

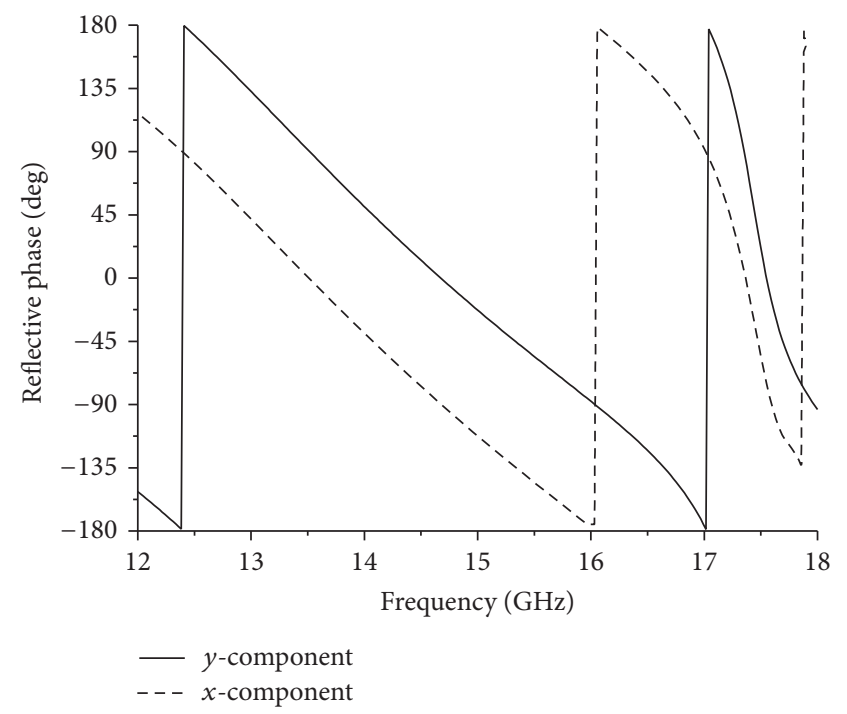

(a)

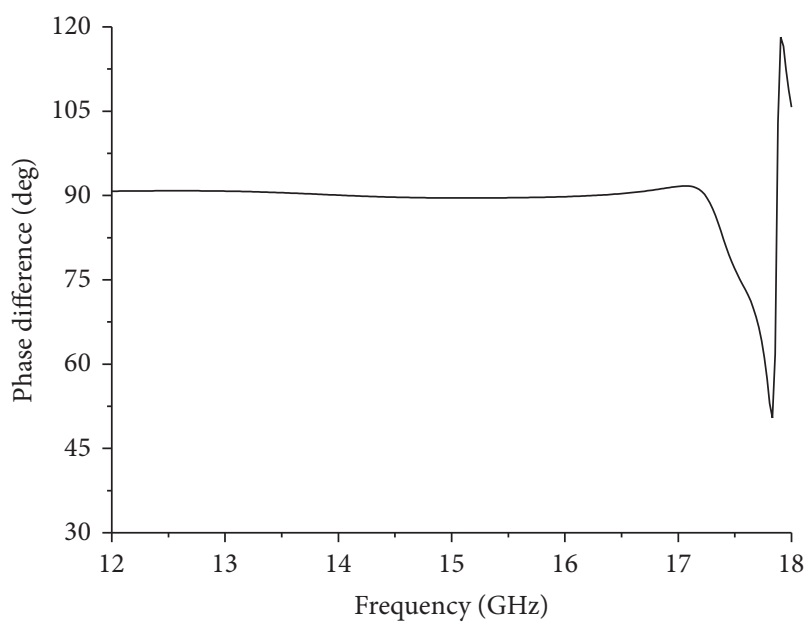

(b)

FIGURE 12: The phase (a) and the phase difference (b) of the $y$ polarized and $x$-polarized reflectance.

Figure 13 shows the calculated ellipticity which fluctuates around $-40 \mathrm{deg}$ and equals $-45 \mathrm{deg}$ at $13.1 \mathrm{GHz}, 15.07 \mathrm{GHz}$, and $16.9 \mathrm{GHz}$.

To further evaluate the circular polarization property of the reflective wave, the axial ratio is calculated by $|A R|_{d B}=$ $20 \lg |\tan \eta|$ and shown in Figure 14, which indicates that the axial ratio is below $3 \mathrm{~dB}$ from $12.66 \mathrm{GHz}$ to $17.43 \mathrm{GHz}$. Thus, the reflected wave can be considered as a left-handed circularly polarized wave.

A sample is fabricated and measured to demonstrate the properties of the circular polarizer as shown in Figure 15. As shown in Figure 15(b), an $x$-polarized horn is used as a source; the $x$ - and $y$-components of the reflective wave are received by the $x$ - and $y$-polarized horns, respectively, with a vector network analyzer (Agilent E8363b). The amplitude and phase of the measured reflectance are shown in Figures 16 and 17. The axial ratio calculated from the measurement results is shown in Figure 18. The measurement results 


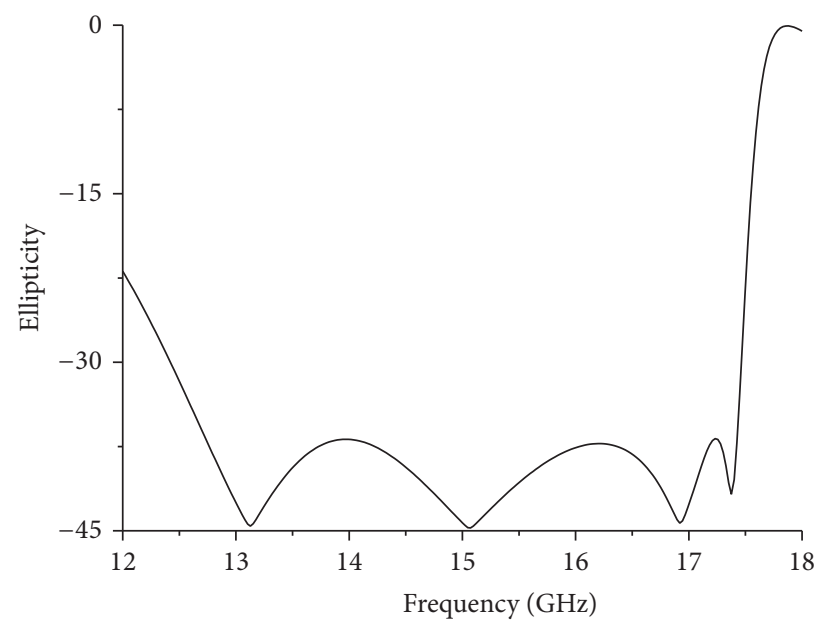

FIGURE 13: The calculated ellipticity.

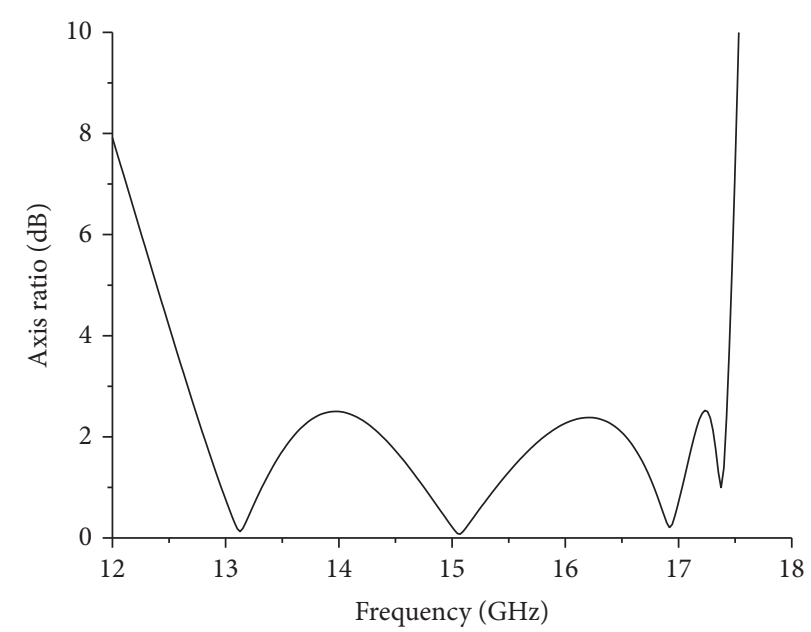

FIgURE 14: The calculated axial ratio.

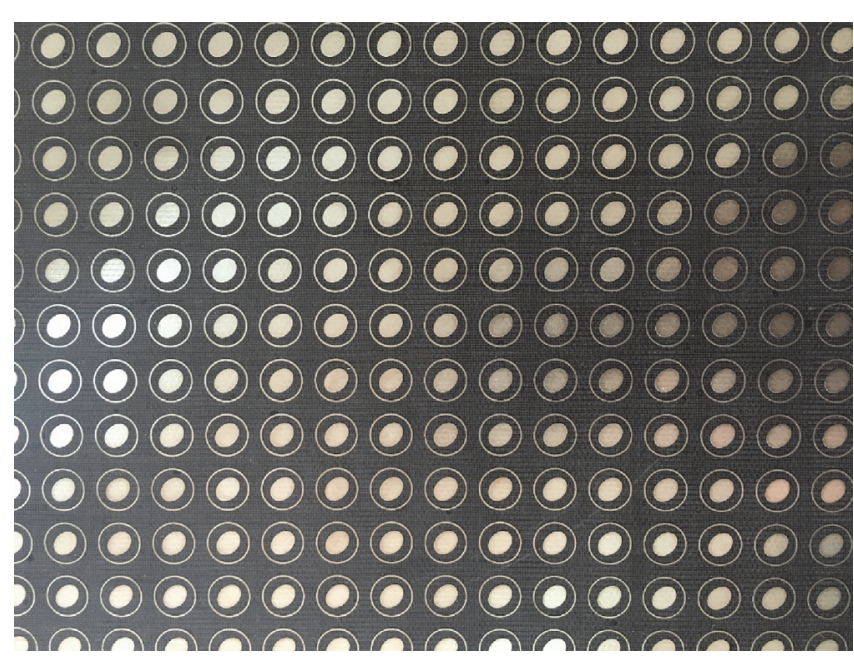

(a)

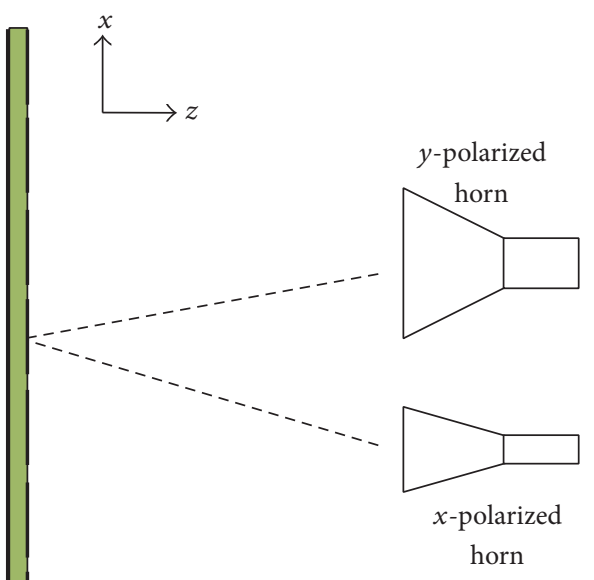

(b)

FIGURE 15: (a) Photo of the fabricated sample; (b) schematic of the measurement. 


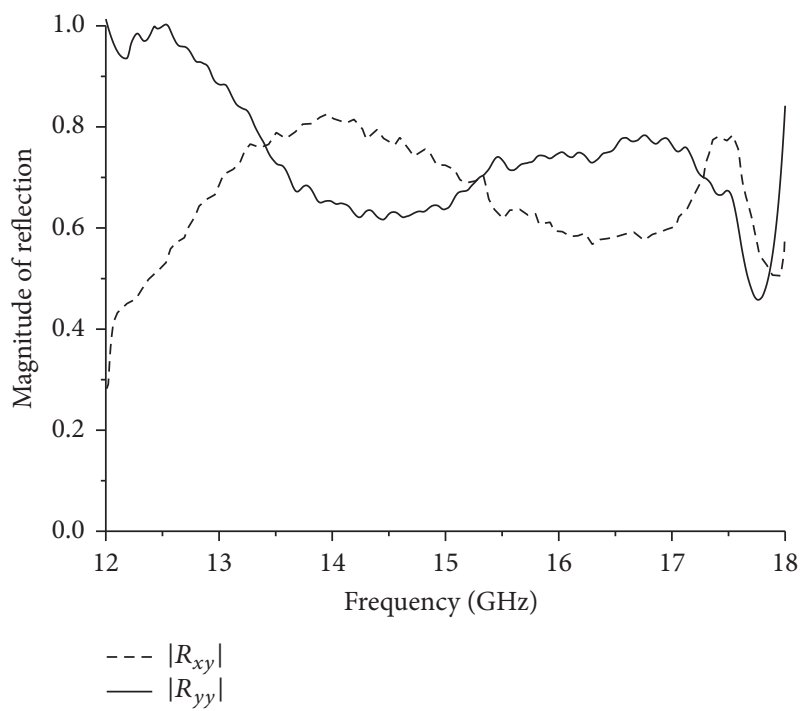

Figure 16: The measured reflectance.

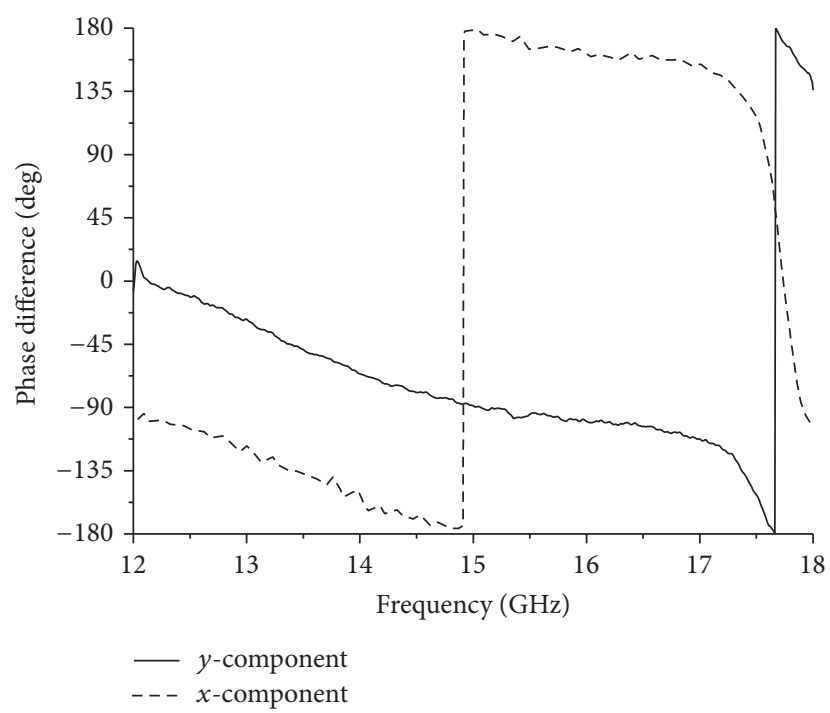

(a)

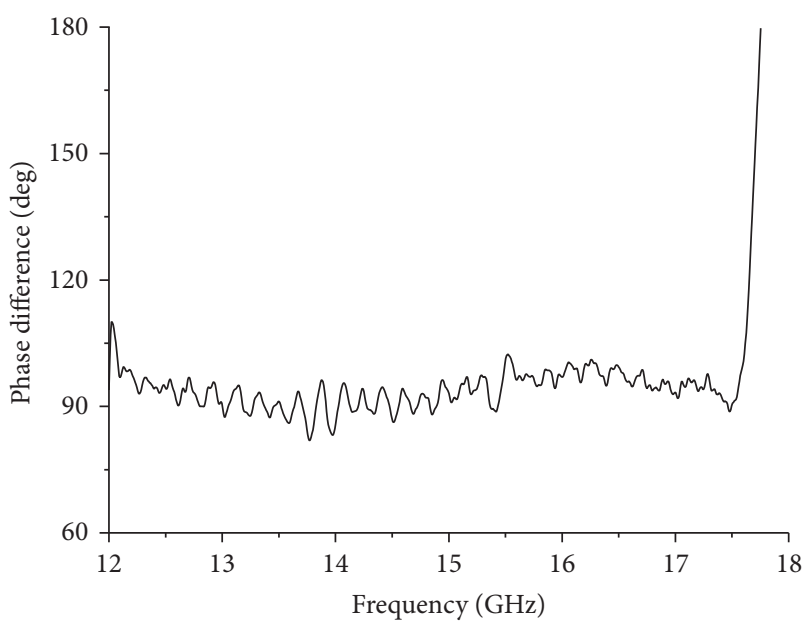

(b)

FIGURE 17: The measured reflectance: (a) phase; (b) phase difference.

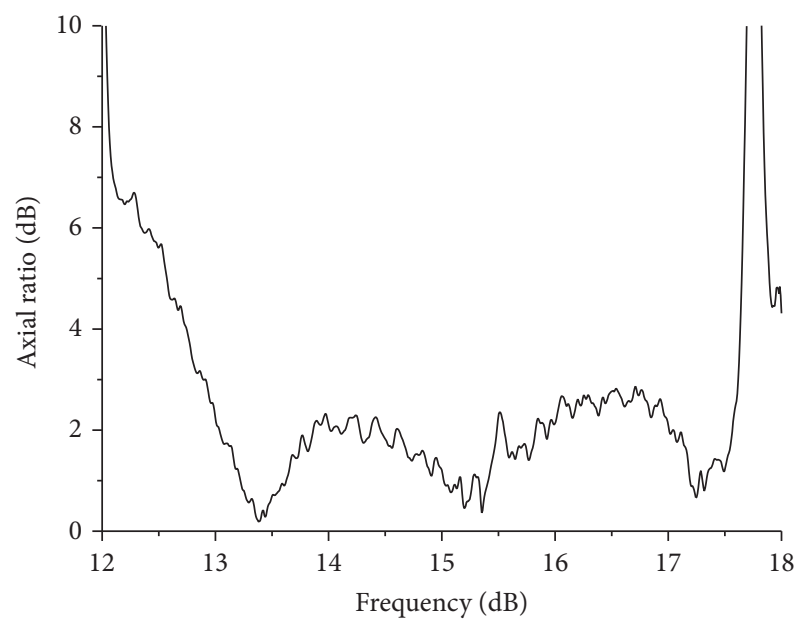

FIgURE 18: The measured axial ratio.

confirm the simulation results, and the differences in the simulation results are caused by the machining error and the measurement error.

\section{Conclusion}

In this paper, a broadband circular polarizer is designed using a ring/disk cavity. Different plasmon hybridizations are excited in the ring/disk cavity at different frequencies, which cause a broadband 90 deg phase difference between the major axis and minor axis polarized reflective waves from $12.66 \mathrm{GHz}$ to $17.43 \mathrm{GHz}$. Thus, a linearly polarized incident wave can be converted to a circularly polarized wave within a bandwidth of about $30 \%$.

\section{Conflicts of Interest}

The authors declare that they have no competing interests.

\section{Acknowledgments}

This work was supported by the National Natural Science Foundation of China (Grant nos. 61501365, 61471292, 61601360, 61471388, and 61331005) and China Postdoctoral Science Foundation (Grant no. 2015M580849).

\section{References}

[1] S. Liu, T. J. Cui, Q. Xu et al., "Anisotropic coding metamaterials and their powerful manipulation of differently polarized terahertz waves," Light: Science and Applications, vol. 5, Article ID e16076, 2016.

[2] S. Liu, Q. Cheng, Q. Xu et al., "Free-standing metasurfaces for high-efficiency transmitarrays for controlling terahertz waves," Advanced Optical Materials, vol. 4, no. 3, pp. 384-390, 2016.

[3] H.-T. Chen, A. J. Taylor, and N. Yu, "A review of metasurfaces: physics and applications," Reports on Progress in Physics, vol. 79, no. 7, Article ID 076401, 2016.

[4] P. Ginzburg, F. J. Rodríguez-Fortuño, A. Martínez, and A. V. Zayats, "Analogue of the quantum hanle effect and polarization 
conversion in non-hermitian plasmonic metamaterials," Nano Letters, vol. 12, no. 12, pp. 6309-6314, 2012.

[5] H. Shi, A. Zhang, S. Zheng, J. Li, and Y. Jiang, "Dual-band polarization angle independent $90 \circ$ polarization rotator using twisted electric-field-coupled resonators," Applied Physics Letters, vol. 104, no. 3, Article ID 034102, 2014.

[6] S. J. Li, X. Y. Cao, L. M. Xu et al., "Ultra-broadband reflective metamaterial with RCS reduction based on polarization convertor, information entropy theory and genetic optimization algorithm," Scientific Reports, vol. 5, article 37409, 2016.

[7] H. Chen, J. Wang, H. Ma et al., "Ultra-wideband polarization conversion metasurfaces based on multiple plasmon resonances," Journal of Applied Physics, vol. 115, no. 15, Article ID 154504, 2014.

[8] T. Tuovinen, E. T. Salonen, and M. Berg, "An artificially anisotropic antenna substrate for the generation of circular polarization," IEEE Transactions on Antennas and Propagation, vol. 64, no. 11, pp. 4937-4942, 2016.

[9] W. Li, S. Xia, B. He et al., "A reconfigurable polarization converter using active metasurface and its application in horn antenna," IEEE Transactions on Antennas and Propagation, vol. 64, no. 12, pp. 5281-5290, 2016.

[10] X. Ma, C. Huang, W. Pan, B. Zhao, J. Cui, and X. Luo, "A dual circularly polarized horn antenna in Ku-band based on chiral metamaterial," IEEE Transactions on Antennas and Propagation, vol. 62, no. 4, pp. 2307-2311, 2014.

[11] H. Shi, J. Chen, A. Zhang, and Y. Jiang, "Design of a circular polarized horn antenna with an anisotropic metamaterial slab," Frequenz, vol. 67, no. 9-10, pp. 271-276, 2013.

[12] F. Yang and Y. Rahmat-Samii, "A low profile single dipole antenna radiating circularly polarized waves," IEEE Transactions on Antennas and Propagation, vol. 53, no. 9, pp. 3083-3086, 2005.

[13] F. Monticone and A. Alù, "Metamaterials and plasmonics: from nanoparticles to nanoantenna arrays, metasurfaces, and metamaterials," Chinese Physics B, vol. 23, no. 4, Article ID 047809, 2014.

[14] B. Luk'Yanchuk, N. I. Zheludev, S. A. Maier et al., “The Fano resonance in plasmonic nanostructures and metamaterials," Nature Materials, vol. 9, no. 9, pp. 707-715, 2010.

[15] V. A. Fedotov, M. Rose, S. L. Prosvirnin, N. Papasimakis, and N. I. Zheludev, "Sharp trapped-mode resonances in planar metamaterials with a broken structural symmetry," Physical Review Letters, vol. 99, no. 14, Article ID 147401, 2007.

[16] G.-X. Dong, H.-Y. Shi, S. Xia et al., "Ultra-broadband and highefficiency polarization conversion metasurface with multiple plasmon resonance modes," Chinese Physics B, vol. 25, no. 8, Article ID 084202, 2016.

[17] F. Wang, A. Chakrabarty, F. Minkowski, K. Sun, and Q.-H. Wei, "Polarization conversion with elliptical patch nanoantennas," Applied Physics Letters, vol. 101, no. 2, Article ID 023101, 2012.

[18] P. V. Tuong, J. W. Park, V. D. Lam, W. H. Jang, S. A. Nikitov, and Y. P. Lee, "Dielectric and Ohmic losses in perfectly absorbingmetamaterials," Optics Communications, vol. 295, pp. 17-20, 2013.

[19] V. A. Podolskiy, A. K. Sarychev, and V. M. Shalaev, "Plasmon modes and negative refraction in metal nanowire composites," Optics Express, vol. 11, no. 7, pp. 735-745, 2003.

[20] H. Lu, X. Liu, D. Mao, and G. Wang, "Plasmonic nanosensor based on Fano resonance in waveguide-coupled resonators," Optics Letters, vol. 37, no. 18, pp. 3780-3782, 2012.
[21] F. Hao, E. M. Larsson, T. A. Ali, D. S. Sutherland, and P. Nordlander, "Shedding light on dark plasmons in gold nanorings," Chemical Physics Letters, vol. 458, no. 4-6, pp. 262-266, 2008.

[22] A. Chakrabarty, F. Wang, F. Minkowski, K. Sun, and Q.-H. Wei, "Cavity modes and their excitations in elliptical plasmonic patch nanoantennas," Optics Express, vol. 20, no. 11, pp. 1161511624, 2012.

[23] Y. Sonnefraud, N. Verellen, H. Sobhani et al., "Experimental realization of subradiant, superradiant, and fano resonances in ring/disk plasmonic nanocavities," ACS Nano, vol. 4, no. 3, pp. 1664-1670, 2010.

[24] F. Hao, Y. Sonnefraud, P. Van Dorpe, S. A. Maier, N. J. Halas, and P. Nordlander, "Symmetry breaking in plasmonic nanocavities: subradiant LSPR sensing and a tunable Fano resonance," Nano Letters, vol. 8, no. 11, pp. 3983-3988, 2008.

[25] H. Shi, J. Li, A. Zhang, J. Wang, and Z. Xu, "Broadband cross polarization converter using plasmon hybridizations in a ring/ disk cavity," Optics Express, vol. 22, no. 17, pp. 20973-20981, 2014.

[26] A. B. Khanikaev, S. H. Mousavi, C. Wu, N. Dabidian, K. B. Alici, and G. Shvets, "Electromagnetically induced polarization conversion," Optics Communications, vol. 285, no. 16, pp. 34233427, 2012.

[27] L. Zhu, F.-Y. Meng, L. Dong, J.-H. Fu, F. Zhang, and Q. $\mathrm{Wu}$, "Polarization manipulation based on electromagnetically induced transparency-like (EIT-like) effect," Optics Express, vol. 21, no. 26, pp. 32099-32110, 2013. 


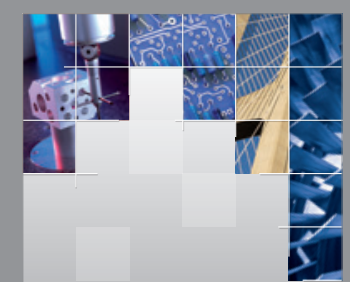

\section{Enfincering}
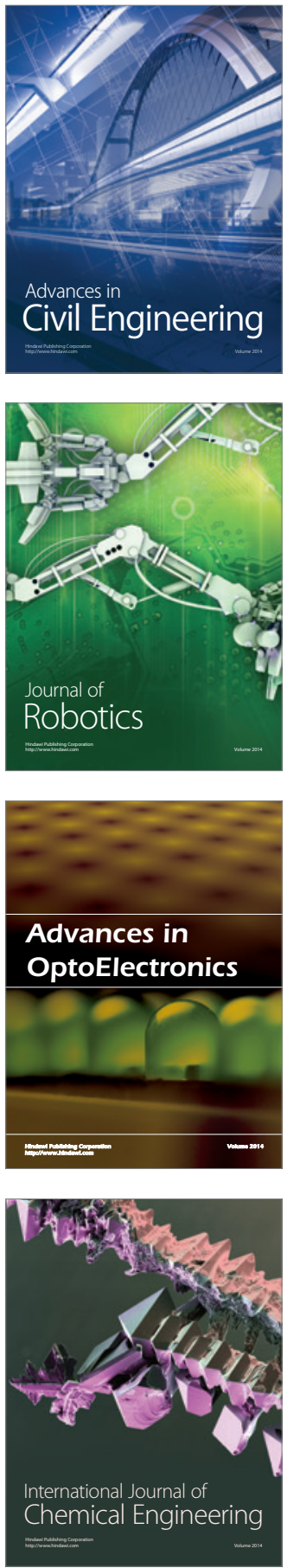

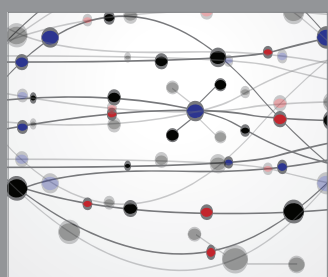

The Scientific World Journal

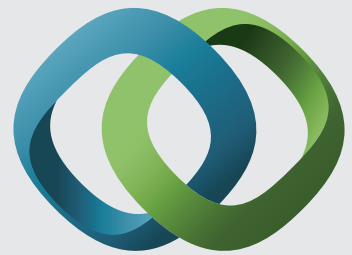

\section{Hindawi}

Submit your manuscripts at

https://www.hindawi.com
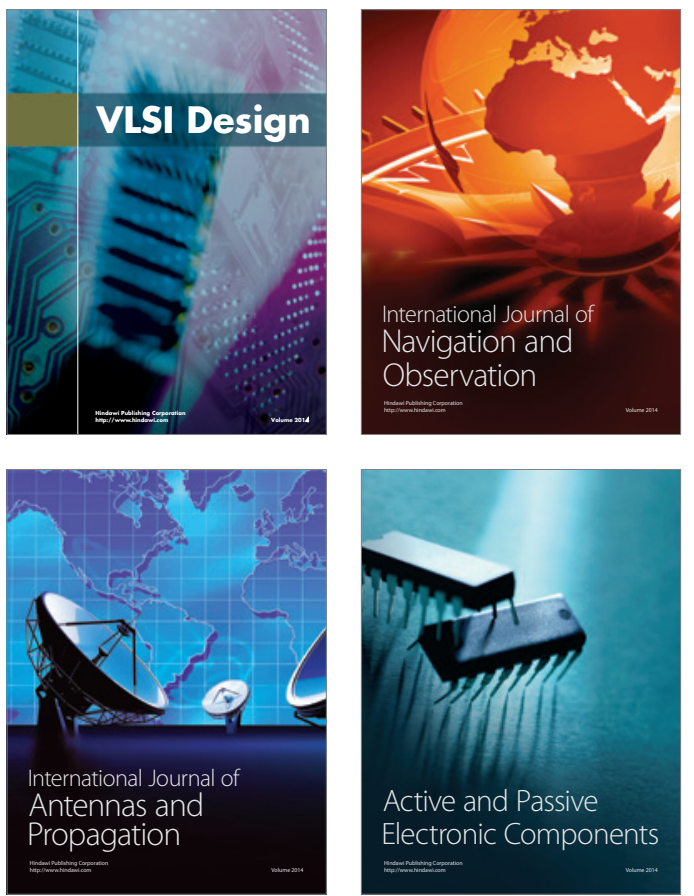
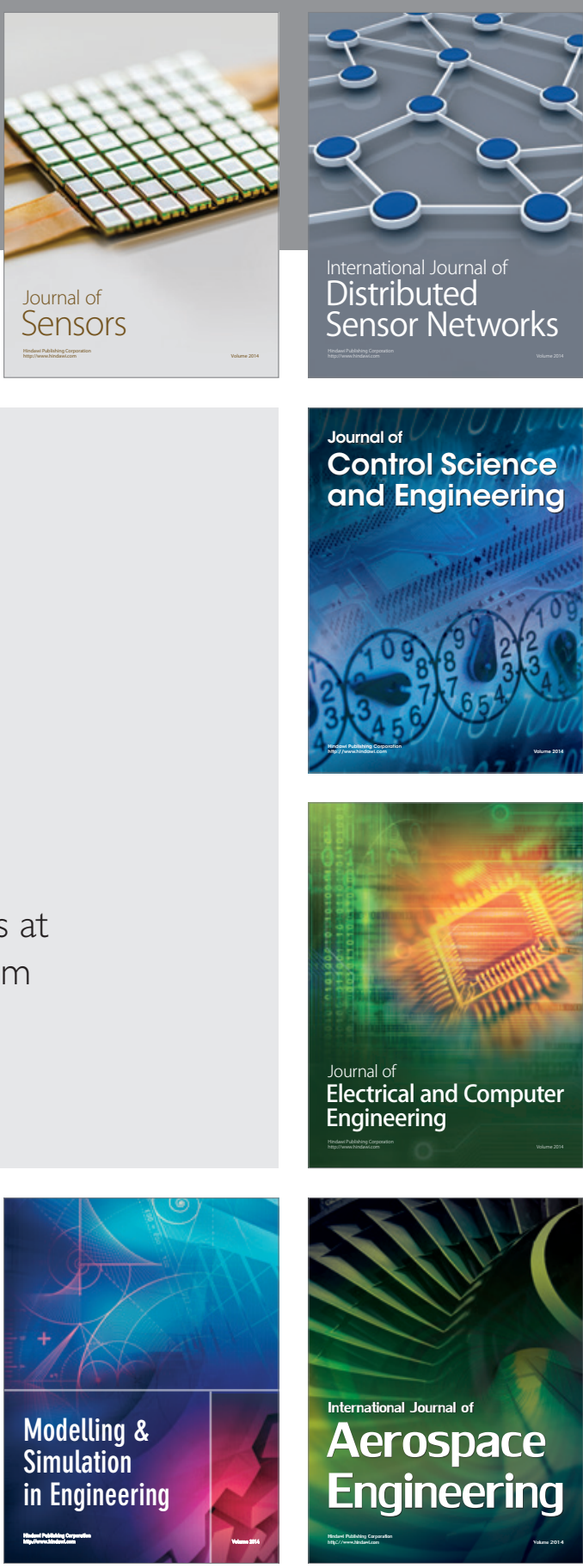

International Journal of

Distributed

Sensor Networks

$-$

Joumal of

Control Science

and Engineering
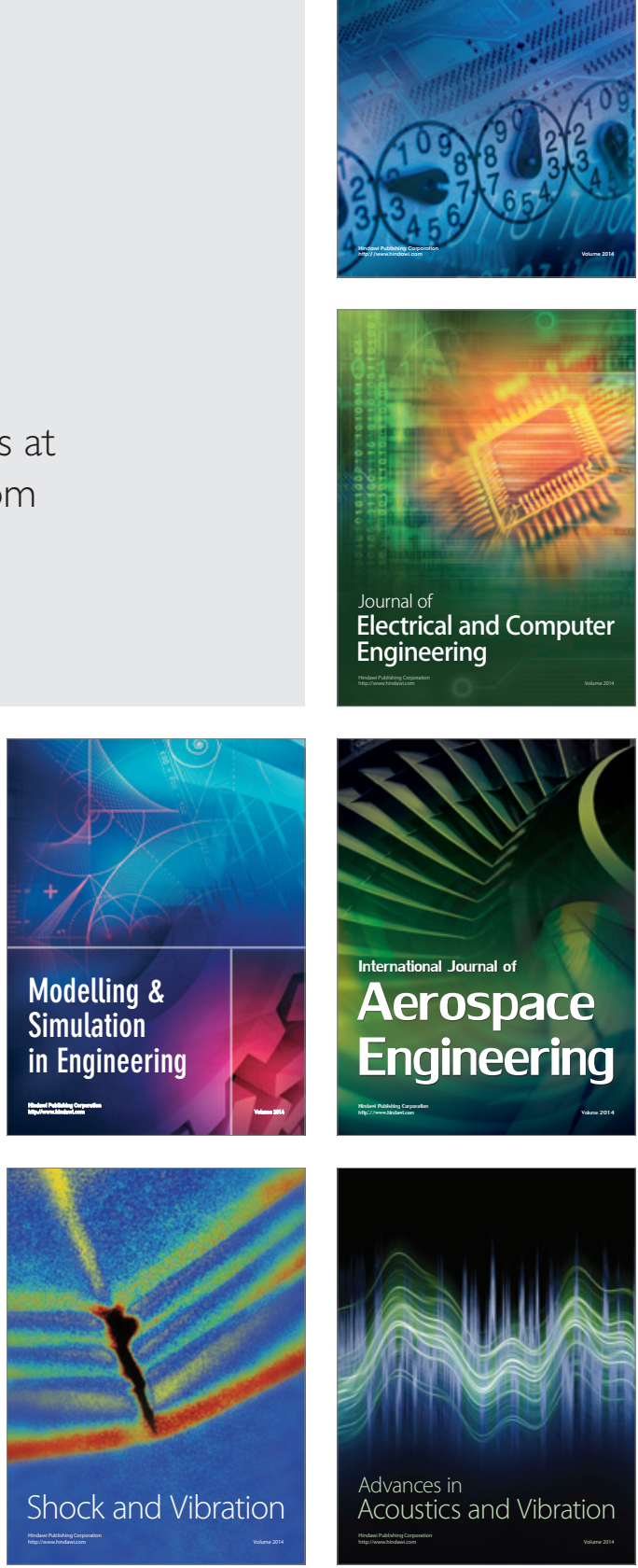